\title{
Sobolev embeddings for Herz-type Triebel-Lizorkin spaces
}

\author{
Douadi Drihem *
}

August 25, 2018

\begin{abstract}
In this paper we prove the Sobolev embeddings for Herz-type Triebel-Lizorkin spaces,

$$
\dot{K}_{q}^{\alpha_{2}, r} F_{\theta}^{s_{2}} \hookrightarrow \dot{K}_{s}^{\alpha_{1}, p} F_{\beta}^{s_{1}}
$$

where the parameters $\alpha_{1}, \alpha_{2}, s_{1}, s_{2}, s, q, r, p, \beta$ and $\theta$ satisfy some suitable conditions. An application we obtain new embeddings between Herz and TriebelLizorkin spaces. Moreover, we present the Sobolev embeddings for TriebelLizorkin spaces equipped with power weights. All these results cover the results on classical Triebel-Lizorkin spaces.
\end{abstract}

MSC 2010: Primary 46E35: Secondary 42B25.

Key Words and Phrases: Triebel-Lizorkin spaces, Herz spaces, Sobolev embedding.

\section{Introduction}

Function spaces have been widely used in various areas of analysis such as harmonic analysis and partial differential equations. In recent years, there has been increasing interest in a new family of function spaces which generalize the Besov spaces and Triebel-Lizorkin spaces. Some example of these spaces can be mentioned such as Herztype Triebel-Lizorkin spaces, $\dot{K}_{q}^{\alpha, p} F_{\beta}^{s}$, that initially appeared in the papers of J. Xu and D. Yang [19], 20] and [21. Several basic properties were established, such as the Fourier analytical characterisation and lifting properties. When $\alpha=0$ and $p=q$ they coincide with the usual function spaces $F_{p, q}^{s}$.

The interest in these spaces comes not only from theoretical reasons but also from their applications to several classical problems in analysis. In [11], Lu and Yang introduced the Herz-type Sobolev and Bessel potential spaces. They gave some applications to partial differential equations. Also in [17], Y. Tsutsui, studied the Cauchy problem for Navier-Stokes equations on Herz spaces and weak Herz spaces.

Since the Sobolev embedding plays an important role in theory of function spaces and PDE's, the main aim of this paper is to prove the Sobolev embedding of $\dot{K}_{q}^{\alpha, p} F_{\beta}^{s}$ spaces.

*M'sila University, Department of Mathematics, Laboratory of Functional Analysis and Geometry of Spaces , P.O. Box 166, M'sila 28000, Algeria, e-mail: douadidr@yahoo.fr 
First we shall prove the Sobolev embeddings of associated sequence spaces. Then, from the so-called $\varphi$-transform characterization in the sense of Frazier and Jawerth, we deduce the main result of this paper. As a consequence, we obtain new JawerthFranke-type embeddings, the Sobolev embeddings for Triebel-Lizorkin spaces equipped with power weights, new embeddings between Herz and Triebel-Lizorkin spaces, and we present some remarks about the wavelet caracterization of Herz-Triebel-Lizorkin spaces. All these results generalize the existing classical results on Triebel-Lizorkin spaces.

To recall the definition of these function spaces, we need some notation. For any $u>0, k \in \mathbb{Z}$ we set $C(u)=\left\{x \in \mathbb{R}^{n}: u / 2 \leq|x|<u\right\}$ and $C_{k}=C\left(2^{k}\right)$. For $x \in \mathbb{R}^{n}$ and $r>0$ we denote by $B(x, r)$ the open ball in $\mathbb{R}^{n}$ with center $x$ and radius $r$. Let $\chi_{k}$, for $k \in \mathbb{Z}$, denote the characteristic function of the set $C_{k}$. The expression $f \approx g$ means that $C g \leq f \leq c g$ for some independent constants $c, C$ and non-negative functions $f$ and $g$.

We denote by $|\Omega|$ the $n$-dimensional Lebesgue measure of $\Omega \subseteq \mathbb{R}^{n}$. For any measurable subset $\Omega \subseteq \mathbb{R}^{n}$ the Lebesgue space $L^{p}(\Omega), 0<p \leq \infty$ consists of all measurable functions for which $\left\|f \mid L^{p}(\Omega)\right\|=\left(\int_{\Omega}|f(x)|^{p} d x\right)^{1 / p}<\infty, 0<p<\infty$ and $\left\|f\left|L^{\infty}(\Omega) \|=\operatorname{ess-sup}\right| f(x) \mid<\infty\right.$. If $\Omega=\mathbb{R}^{n}$ we put $L^{p}\left(\mathbb{R}^{n}\right)=L^{p}$ and $\left\|f \mid L^{p}\left(\mathbb{R}^{n}\right)\right\|=\|f\|_{p}$. The Hardy-Littlewood maximal operator $\mathcal{M}$ is defined on locally integrable functions by

$$
\mathcal{M} f(x)=\sup _{r>0} \frac{1}{|B(x, r)|} \int_{B(x, r)}|f(y)| d y
$$

and $\mathcal{M}_{t} f=\left(\mathcal{M}|f|^{t}\right)^{1 / t}$ for any $0<t \leq 1$. The symbol $\mathcal{S}\left(\mathbb{R}^{n}\right)$ is used in place of the set of all Schwartz functions $\varphi$ on $\mathbb{R}^{n}$ and we denote by $\mathcal{S}^{\prime}\left(\mathbb{R}^{n}\right)$ the dual space of all tempered distributions on $\mathbb{R}^{n}$. We define the Fourier transform of a function $f \in \mathcal{S}\left(\mathbb{R}^{n}\right)$ by $\mathcal{F}(f)(\xi)=(2 \pi)^{-n / 2} \int_{\mathbb{R}^{n}} e^{-i x \cdot \xi} f(x) d x$. Its inverse is denoted by $\mathcal{F}^{-1} f$. Both $\mathcal{F}$ and $\mathcal{F}^{-1}$ are extended to the dual Schwartz space $\mathcal{S}^{\prime}\left(\mathbb{R}^{n}\right)$ in the usual way.

Let $\mathbb{Z}^{n}$ be the lattice of all points in $\mathbb{R}^{n}$ with integer-valued components. If $v \in \mathbb{N}_{0}$ and $m=\left(m_{1}, \ldots, m_{n}\right) \in \mathbb{Z}^{n}$ we denote $Q_{v, m}$ the dyadic cube in $\mathbb{R}^{n}$

$$
Q_{v, m}=\left\{\left(x_{1}, \ldots, x_{n}\right): m_{i} \leq 2^{v} x_{i}<m_{i}+1, i=1,2, \ldots, n\right\} .
$$

By $\chi_{v, m}$ we denote the characteristic function of the cube $Q_{v, m}$.

Given two quasi-Banach spaces $X$ and $Y$, we write $X \hookrightarrow Y$ if $X \subset Y$ and the natural embedding of $X$ in $Y$ is continuous. We use $c$ as a generic positive constant, i.e. a constant whose value may change from appearance to appearance.

\section{Function spaces}

We start by recalling the definition and some of the properties of the homogenous Herz spaces $\dot{K}_{q}^{\alpha, p}$.

Definition 1 Let $\alpha \in \mathbb{R}, 0<p, q \leq \infty$. The homogeneous Herz space $\dot{K}_{q}^{\alpha, p}$ is defined by

$$
\dot{K}_{q}^{\alpha, p}=\left\{f \in L_{\mathrm{loc}}^{q}\left(\mathbb{R}^{n} \backslash\{0\}\right):\left\|f \mid \dot{K}_{q}^{\alpha, p}\right\|<\infty\right\}
$$


where

$$
\left\|f \mid \dot{K}_{q}^{\alpha, p}\right\|=\left(\sum_{k=-\infty}^{\infty} 2^{k \alpha p}\left\|f \chi_{k}\right\|_{q}^{p}\right)^{1 / p}
$$

with the usual modifications made when $p=\infty$ and/or $q=\infty$.

The spaces $\dot{K}_{q}^{\alpha, p}$ are quasi-Banach spaces and if $\min (p, q) \geq 1$ then $\dot{K}_{q}^{\alpha, p}$ are Banach spaces. When $\alpha=0$ and $0<p=q \leq \infty$ then $\dot{K}_{p}^{0, p}$ coincides with the Lebesgue spaces $L^{p}$. Various important results have been proved in the space $\dot{K}_{q}^{\alpha, p}$ under some assumptions on $\alpha, p$ and $q$. The conditions $-\frac{n}{q}<\alpha<n\left(1-\frac{1}{q}\right), 1<q<\infty$ and $0<p \leq \infty$ is crucial in the study of the boundedness of classical operators in $\dot{K}_{q}^{\alpha, p}$ spaces. This fact was first realized by Li and Yang [8] with the proof of the boundedness of the maximal function. The proof of the main result of this section is based on the following result, see Tang and Yang [13].

Lemma 1 Let $1<\beta<\infty, 1<q<\infty$ and $0<p \leq \infty$. If $\left\{f_{j}\right\}_{j=0}^{\infty}$ is a sequence of locally integrable functions on $\mathbb{R}^{n}$ and $-\frac{n}{q}<\alpha<n\left(1-\frac{1}{q}\right)$, then

$$
\left\|\left(\sum_{j=0}^{\infty}\left(\mathcal{M} f_{j}\right)^{\beta}\right)^{1 / \beta}\left|\dot{K}_{q}^{\alpha, p}\|\leq c\|\left(\sum_{j=0}^{\infty}\left|f_{j}\right|^{\beta}\right)^{1 / \beta}\right| \dot{K}_{q}^{\alpha, p}\right\| .
$$

A detailed discussion of the properties of these spaces my be found in the papers [7], [9], [10], and references therein.

Now, we present the Fourier analytical definition of Herz-type Triebel-Lizorkin spaces $\dot{K}_{q}^{\alpha, p} F_{\beta}^{s}$ and recall their basic properties. We first need the concept of a smooth dyadic resolution of unity. Let $\phi_{0}$ be a function in $\mathcal{S}\left(\mathbb{R}^{n}\right)$ satisfying $\phi_{0}(x)=1$ for $|x| \leq 1$ and $\phi_{0}(x)=0$ for $|x| \geq 2$. We put $\phi_{j}(x)=\phi_{0}\left(2^{-j} x\right)-\phi_{0}\left(2^{1-j} x\right)$ for $j=1,2,3, \ldots$. Then $\left\{\phi_{j}\right\}_{j \in \mathbb{N}_{0}}$ is a resolution of unity, $\sum_{j=0}^{\infty} \phi_{j}(x)=1$ for all $x \in \mathbb{R}^{n}$. Thus we obtain the Littlewood-Paley decomposition $f=\sum_{j=0}^{\infty} \mathcal{F}^{-1} \phi_{j} * f$ of all $f \in \mathcal{S}^{\prime}\left(\mathbb{R}^{n}\right)$ (convergence in $\left.\mathcal{S}^{\prime}\left(\mathbb{R}^{n}\right)\right)$.

We are now in a position to state the definition of Herz-type Triebel-Lizorkin spaces.

Definition 2 Let $\alpha, s \in \mathbb{R}, 0<p, q<\infty$ and $0<\beta \leq \infty$. The Herz-type TriebelLizorkin space $\dot{K}_{q}^{\alpha, p} F_{\beta}^{s}$ is the collection of all $f \in \mathcal{S}^{\prime}\left(\mathbb{R}^{n}\right)$ such that

$$
\left\|f\left|\dot{K}_{q}^{\alpha, p} F_{\beta}^{s}\|=\|\left(\sum_{j=0}^{\infty} 2^{j s \beta}\left|\mathcal{F}^{-1} \phi_{j} * f\right|^{\beta}\right)^{1 / \beta}\right| \dot{K}_{q}^{\alpha, p}\right\|<\infty,
$$

with the obvious modification if $\beta=\infty$.

Remark 1 Let $s \in \mathbb{R}, 0<p, q<\infty, 0<\beta \leq \infty$ and $\alpha>-n / q$. The spaces $\dot{K}_{q}^{\alpha, p} F_{\beta}^{s}$ are independent of the particular choice of the smooth dyadic resolution of unity $\left\{\phi_{j}\right\}_{j \in \mathbb{N}_{0}}$ (in the sense of equivalent quasi-norms). In particular $\dot{K}_{q}^{\alpha, p} F_{\beta}^{s}$ are quasi-Banach spaces and if $p, q, \beta \geq 1$, then $\dot{K}_{q}^{\alpha, p} F_{\beta}^{s}$ are Banach spaces. Further results, concerning, for instance, lifting properties, Fourier multiplier and local means characterizations can be found in [20, 21], 22] and [24]. 
Now we give the definitions of the spaces $B_{p, \beta}^{s}$ and $F_{p, \beta}^{s}$.

Definition 3 (i) Let $s \in \mathbb{R}$ and $0<p, \beta \leq \infty$. The Besov space $B_{p, \beta}^{s}$ is the collection of all $f \in \mathcal{S}^{\prime}\left(\mathbb{R}^{n}\right)$ such that

$$
\left\|f \mid B_{p, \beta}^{s}\right\|=\left(\sum_{j=0}^{\infty} 2^{j s \beta}\left\|\mathcal{F}^{-1} \phi_{j} * f\right\|_{p}^{\beta}\right)^{1 / \beta}<\infty .
$$

(ii) Let $s \in \mathbb{R}, 0<p<\infty$ and $0<\beta \leq \infty$. The Triebel-Lizorkin space $F_{p, \beta}^{s}$ is the collection of all $f \in \mathcal{S}^{\prime}\left(\mathbb{R}^{n}\right)$ such that

$$
\left\|f \mid F_{p, \beta}^{s}\right\|=\left\|\left(\sum_{j=0}^{\infty} 2^{j s \beta}\left|\mathcal{F}^{-1} \phi_{j} * f\right|^{\beta}\right)^{1 / \beta}\right\|_{p}<\infty .
$$

The theory of the spaces $B_{p, \beta}^{s}$ and $F_{p, \beta}^{s}$ has been developed in detail in [14], 15] and [16] but has a longer history already including many contributors; we do not want to discuss this here. Clearly, for $s \in \mathbb{R}, 0<p<\infty$ and $0<\beta \leq \infty$,

$$
\dot{K}_{p}^{0, p} F_{\beta}^{s}=F_{p, \beta}^{s} .
$$

Let us consider $k_{0}, k \in \mathcal{S}\left(\mathbb{R}^{n}\right)$ and $S \geq-1$ an integer such that for an $\varepsilon>0$

$$
\begin{array}{rll}
\left|\mathcal{F} k_{0}(\xi)\right|>0 & \text { for } & |\xi|<2 \varepsilon \\
|\mathcal{F} k(\xi)|>0 & \text { for } & \frac{\varepsilon}{2}<|\xi|<2 \varepsilon
\end{array}
$$

and

$$
\int_{\mathbb{R}^{n}} x^{\alpha} k(x) d x=0 \quad \text { for any } \quad|\alpha| \leq S .
$$

Here (21) and (3) are Tauberian conditions, while (41) are moment conditions on $k$. We recall the notation

$$
k_{t}(x)=t^{-n} k\left(t^{-1} x\right), \quad k_{j}(x)=k_{2^{-j}}(x), \quad \text { for } \quad t>0 \quad \text { and } \quad j \in \mathbb{N} .
$$

Usually $k_{j} * f$ is called local mean. The following result is from [23, Theorem 1].

Theorem 1 Let $\alpha, s \in \mathbb{R}, 0<p, q<\infty, 0<\beta \leq \infty, \alpha>-n / q$ and $s<S+1$. Then

$$
\left\|f\left|\dot{K}_{q}^{\alpha, p} F_{\beta}^{s}\left\|^{\prime}=\right\|\left(2^{j s} k_{j} * f\right)_{j}\right| \dot{K}_{q}^{\alpha, p}\left(\ell_{\beta}\right)\right\|,
$$

is equivalent quasi-norm on $\dot{K}_{q}^{\alpha, p} F_{\beta}^{s}$.

We introduce the sequence spaces associated with the function spaces $\dot{K}_{q}^{\alpha, p} F_{\beta}^{s}$. If

$$
\lambda=\left\{\lambda_{v, m} \in \mathbb{C}: v \in \mathbb{N}_{0}, m \in \mathbb{Z}^{n}\right\},
$$

$\alpha, s \in \mathbb{R}, 0<p, q<\infty$ and $0<\beta \leq \infty$, we set

$$
\left\|\lambda\left|\dot{K}_{q}^{\alpha, p} f_{\beta}^{s}\|=\|\left(\sum_{v=0}^{\infty} \sum_{m \in \mathbb{Z}^{n}} 2^{v s \beta}\left|\lambda_{v, m}\right|^{\beta} \chi_{v, m}\right)^{1 / \beta}\right| \dot{K}_{q}^{\alpha, p}\right\| .
$$


Let $\Phi, \psi, \varphi$ and $\Psi$ satisfy

$$
\Phi, \Psi, \varphi, \psi \in \mathcal{S}\left(\mathbb{R}^{n}\right)
$$

$$
\operatorname{supp} \mathcal{F} \Phi, \operatorname{supp} \mathcal{F} \Psi \subset \overline{B(0,2)} \text { such that }|\mathcal{F} \Phi(\xi)|,|\mathcal{F} \Psi(\xi)| \geq c \text { if }|\xi| \leq \frac{5}{3}
$$

and

$$
\operatorname{supp} \mathcal{F} \varphi, \operatorname{supp} \mathcal{F} \psi \subset \overline{B(0,2)} \backslash B(0,1 / 2) \text { such that }|\mathcal{F} \varphi(\xi)|,|\mathcal{F} \psi(\xi)| \geq c \text { if } \frac{3}{5} \leq|\xi| \leq \frac{5}{3}
$$

such that

$$
\overline{\mathcal{F} \Phi(-\xi)} \mathcal{F} \Psi(\xi)+\sum_{j=1}^{\infty} \overline{\mathcal{F} \varphi\left(-2^{-j} \xi\right)} \mathcal{F} \psi\left(2^{-j} \xi\right)=1, \quad \xi \in \mathbb{R}^{n},
$$

where $c>0$. Recall that the $\varphi$-transform $S_{\varphi}$ is defined by setting $\left(S_{\varphi} f\right)_{0, m}=\left\langle f, \Phi_{m}\right\rangle$ where $\Phi_{m}(x)=\Phi(x-m)$ and $\left(S_{\varphi} f\right)_{v, m}=\left\langle f, \varphi_{v, m}\right\rangle$ where $\varphi_{v, m}(x)=2^{v n / 2} \varphi\left(2^{v} x-m\right)$ and $v \in \mathbb{N}$. The inverse $\varphi$-transform $T_{\psi}$ is defined by

$$
T_{\psi} \lambda=\sum_{m \in \mathbb{Z}^{n}} \lambda_{0, m} \Psi_{m}+\sum_{v=1}^{\infty} \sum_{m \in \mathbb{Z}^{n}} \lambda_{v, m} \psi_{v, m}
$$

where $\lambda=\left\{\lambda_{v, m} \in \mathbb{C}: v \in \mathbb{N}_{0}, m \in \mathbb{Z}^{n}\right\}$, see [3].

For a sequence $\lambda=\left\{\lambda_{v, m} \in \mathbb{C}: v \in \mathbb{N}_{0}, m \in \mathbb{Z}^{n}\right\}, 0<r \leq \infty$ and a fixed $d>0$, set

$$
\lambda_{v, m, r, d}^{*}=\left(\sum_{h \in \mathbb{Z}^{n}} \frac{\left|\lambda_{v, h}\right|^{r}}{\left(1+2^{v}\left|2^{-v} h-2^{-v} m\right|\right)^{d}}\right)^{1 / r}
$$

and $\lambda_{r, d}^{*}=\left\{\lambda_{v, m, r, d}^{*} \in \mathbb{C}: v \in \mathbb{N}_{0}, m \in \mathbb{Z}^{n}\right\}$.

Lemma 2 Let $\alpha, s \in \mathbb{R}, 0<p \leq \infty, 0<q<\infty, 0<\beta \leq \infty, d>n$ and $\alpha>-n / q$. Then

$$
\left\|\lambda_{\min \left(q, \frac{n}{\frac{m}{q}+\alpha}, \beta\right), d}^{*}\left|\dot{K}_{q}^{\alpha, p} f_{\beta}^{s}\|\approx\| \lambda\right| \dot{K}_{q}^{\alpha, p} f_{\beta}^{s}\right\|
$$

Proof. Obviously,

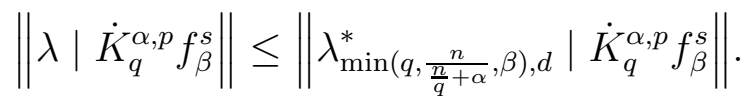

From Lemma A.2 of [3], we obtain

$$
\lambda_{v, m, \min \left(q, \frac{n}{\frac{n}{q}+\alpha}, \beta\right), d}^{*} \leq c \mathcal{M}_{a}\left(\sum_{h \in \mathbb{Z}^{n}}\left|\lambda_{v, h}\right| \chi_{v, h}\right)(x), \quad x \in Q_{v, m},
$$

where,

$$
0<a \leq r=\min \left(q, \frac{n}{\frac{n}{q}+\alpha}, \beta\right)<\infty, d a>n r
$$

and $c>0$ depend only $n$ and $d$. Let $\varepsilon=\frac{d}{n}-1>0$ and $a=\frac{r}{1+\varepsilon / 2}$, then $0<a<r$ and $d a>n r$. Hence

$$
\begin{aligned}
&\left.\| \lambda_{\min \left(q, \frac{n}{q}+\alpha\right.}^{*}, \beta\right), d \\
& \leq c \|\left(\sum_{v=0}^{\infty} \mathcal{K}_{q / \beta}^{\alpha, p} f_{\beta}^{s} \|\right. \\
&\left.\left.\leq \sum_{h \in \mathbb{Z}^{n}} 2^{v s \beta}\left|\lambda_{v, h}\right|^{\beta} \chi_{v, h}\right)\right)^{a / \beta} \mid \dot{K}_{q / a}^{\alpha a, p / a} \|^{1 / a} .
\end{aligned}
$$


Observe that $\frac{\beta}{a}>1, \frac{q}{a}>1$ and $\frac{-n a}{q}<\alpha a<n\left(1-\frac{a}{q}\right)$. Applying Lemma 1 to estimate the last expression by

$$
c\left\|\left(\sum_{v=0}^{\infty} \sum_{h \in \mathbb{Z}^{n}} 2^{v s \beta}\left|\lambda_{v, h}\right|^{\beta} \chi_{v, h}\right)^{1 / \beta}\left|\dot{K}_{q}^{\alpha, p}\|=c\| \lambda\right| \dot{K}_{q}^{\alpha, p} f_{\beta}^{s}\right\| .
$$

The proof of the lemma is thus complete.

To prove the main results of this paper we need the following theorem.

Theorem 2 Let $\alpha, s \in \mathbb{R}, 0<p<\infty, 0<q<\infty, 0<\beta \leq \infty$ and $\alpha>-n / q$. Suppose that $\varphi$ and $\Phi$ satisfy (7)-(10). The operators $S_{\varphi}: \dot{K}_{q}^{\alpha, p} F_{\beta}^{s} \rightarrow \dot{K}_{q}^{\alpha, p} f_{\beta}^{s}$ and $T_{\psi}: \dot{K}_{q}^{\alpha, p} f_{\beta}^{s} \rightarrow \dot{K}_{q}^{\alpha, p} F_{\beta}^{s}$ are bounded. Furthermore, $T_{\psi} \circ S_{\varphi}$ is the identity on $\dot{K}_{q}^{\alpha, p} F_{\beta}^{s}$.

Proof. We use the same arguments of [3, Theorem 2.2], see also [25, Theorem 2.1] and [1, Theorem 3.12]. For any $f \in \mathcal{S}^{\prime}\left(\mathbb{R}^{n}\right)$ we put $\sup (f)=\left\{\sup _{v, m}(f): v \in \mathbb{N}_{0}, m \in \mathbb{Z}^{n}\right\}$ where

$$
\sup _{v, m}(f)=\sup _{y \in Q_{v, m}}\left|\widetilde{\varphi_{v}} * f(y)\right|
$$

if $v \in \mathbb{N}, m \in \mathbb{Z}^{n}$ and

$$
\sup _{0, m}(f)=\sup _{y \in Q_{0, m}}|\widetilde{\Phi} * f(y)|
$$

if $m \in \mathbb{Z}^{n}$. For any $\gamma \in \mathbb{Z}_{+}$, we define the sequence $\inf _{\gamma}(f)=\left\{\inf _{v, m, \gamma}(f): v \in \mathbb{N}_{0}, m \in\right.$ $\left.\mathbb{Z}^{n}\right\}$ by setting

$$
\inf _{v, m, \gamma}(f)=\sup _{h \in \mathbb{Z}^{n}}\left\{\inf _{y \in Q_{v+\gamma, h}}\left|\widetilde{\varphi_{v}} * f(y)\right|: Q_{v+\gamma, h} \cap Q_{v, m} \neq \emptyset\right\}
$$

if $v \in \mathbb{N}, m \in \mathbb{Z}^{n}$ and

$$
\inf _{0, m, \gamma}(f)=\sup _{h \in \mathbb{Z}^{n}}\left\{\inf _{y \in Q_{\gamma, h}}|\widetilde{\Phi} * f(y)|: Q_{\gamma, h} \cap Q_{0, m} \neq \emptyset\right\}
$$

if $m \in \mathbb{Z}^{n}$. Here $\widetilde{\varphi_{j}}(x)=2^{j n} \overline{\varphi\left(-2^{j} x\right)}$ and $\widetilde{\Phi}(x)=\overline{\Phi(-x)}$. As in Lemma A.5 of [3], see also [1] and [25] we obtain

$$
\left\|\inf _{\gamma}(f)\left|\dot{K}_{q}^{\alpha, p} f_{\beta}^{s}\|\leq c\| f\right| \dot{K}_{q}^{\alpha, p} F_{\beta}^{s}\right\|
$$

for any $s \in \mathbb{R}, 0<p, q<\infty, 0<\beta \leq \infty, \alpha>-n / q$ and $\gamma>0$ sufficiently large. Indeed, we have

$$
\left\|\inf _{\gamma}(f)\left|\dot{K}_{q}^{\alpha, p} f_{\beta}^{s}\|=c\|\left(\sum_{m \in \mathbb{Z}^{n}} 2^{j s} \inf _{j-\gamma, m, \gamma}(f) \chi_{j-\gamma, m}\right)_{j \geq \gamma}\right| \dot{K}_{q}^{\alpha, p}\left(\ell_{\beta}\right)\right\|
$$

Define a sequence $\left\{\lambda_{i, k}\right\}_{i \in \mathbb{N}_{0}, k \in \mathbb{Z}^{n}}$ by setting $\lambda_{i, k}=\inf _{y \in Q_{i, k}}\left|\widetilde{\varphi_{i-\gamma}} * f(y)\right|$ and $\lambda_{0, k}=$ $\inf _{y \in Q_{\gamma, k}}|\widetilde{\Phi} * f(y)|$. We have

$$
\inf _{j-\gamma, m, \gamma}(f)=\sup _{h \in \mathbb{Z}^{n}}\left\{\lambda_{j, h}: Q_{j, h} \cap Q_{j-\gamma, m} \neq \emptyset\right\}
$$

and

$$
\inf _{0, m, \gamma}(f)=\sup _{h \in \mathbb{Z}^{n}}\left\{\lambda_{0, h}: Q_{\gamma, h} \cap Q_{0, m} \neq \emptyset\right\}
$$


Let $h \in \mathbb{Z}^{n}$ with $Q_{j, h} \cap Q_{j-\gamma, m} \neq \emptyset$ and $j \geq \gamma$. Then

$$
\lambda_{j, h} \leq c 2^{\gamma d / r} \lambda_{j, z, r, \gamma}^{*}, j>\gamma \quad \text { and } \quad \lambda_{0, h} \leq c 2^{\gamma d / r} \lambda_{0, z, r, \gamma}^{*}, \quad j=\gamma
$$

for any $z \in \mathbb{Z}^{n}$ with $Q_{j, z} \cap Q_{j-\gamma, m} \neq \emptyset$, where the constant $c>0$ does not depend on $j, h$ and $z$. Indeed, we observe

$$
\lambda_{j, h}=\frac{\lambda_{j, h}}{\left(1+2^{j}\left|2^{-j} h-2^{-j} z\right|\right)^{d}}\left(1+2^{j}\left|2^{-j} h-2^{-j} z\right|\right)^{d} .
$$

Let $x \in Q_{j, h} \cap Q_{j-\gamma, m}$ and $y \in Q_{j, z} \cap Q_{j-\gamma, m}$. We have

$$
\left|2^{-j} h-2^{-j} z\right| \leq\left|2^{-j} h-x\right|+|x-y|+\left|y-2^{-j} z\right| \lesssim 2^{2-j}+2^{\gamma-j} .
$$

This implies (11). Hence

$$
\sum_{m \in \mathbb{Z}^{n}} \inf _{j-\gamma, m, \gamma}(f) \chi_{j-\gamma, m} \leq c \sum_{k \in \mathbb{Z}^{n}} \lambda_{j, k, r, d}^{*} \chi_{j, k}, \quad j>\gamma
$$

and

$$
\sum_{m \in \mathbb{Z}^{n}} \inf _{0, m, \gamma}(f) \chi_{0, m} \leq c \sum_{k \in \mathbb{Z}^{n}} \lambda_{0, k, r, d}^{*} \chi_{\gamma, k}, \quad j=\gamma,
$$

with $r=\min \left(q, \frac{n}{\frac{n}{q}+\alpha}, \beta\right)$ and $d>n$. Therefore,

$$
\left\|\inf _{\gamma}(f)\left|\dot{K}_{q}^{\alpha, p} f_{\beta}^{s}\|\leq c\|\left(\sum_{k \in \mathbb{Z}^{n}} 2^{j s} \lambda_{j, k, r, d}^{*} \chi_{j, k}\right)_{j \geq \gamma}\right| \dot{K}_{q}^{\alpha, p}\left(\ell_{\beta}\right)\right\| .
$$

Notice that if $j=\gamma$ we replace $\lambda_{j, k, r, d}^{*} \chi_{j, k}$ by $\lambda_{0, k, r, d}^{*} \chi_{\gamma, k}$. Applying Lemma 2 to estimate this term by

$$
c\left\|\left(\sum_{k \in \mathbb{Z}^{n}} 2^{j s} \lambda_{j, k} \chi_{j, k}\right)_{j>\gamma}\left|\dot{K}_{q}^{\alpha, p}\left(\ell_{\beta}\right)\|+c\| \sum_{k \in \mathbb{Z}^{n}} \lambda_{0, k} \chi_{\gamma, k}\right| \dot{K}_{q}^{\alpha, p}\right\|,
$$

wich is bounded by

$$
c\left\|\left(2^{j s} \widetilde{\varphi_{j-\gamma}} * f\right)_{j>\gamma}\left|\dot{K}_{q}^{\alpha, p}\left(\ell_{\beta}\right)\|+c\| \widetilde{\Phi} * f\right| \dot{K}_{q}^{\alpha, p}\right\| .
$$

By Theorem 1, we obtain

$$
\left\|\inf _{\gamma}(f)\left|\dot{K}_{q}^{\alpha, p} f_{\beta}^{s}\|\leq c\| f\right| \dot{K}_{q}^{\alpha, p} F_{\beta}^{s}\right\|^{\prime} \leq c\left\|f \mid \dot{K}_{q}^{\alpha, p} F_{\beta}^{s}\right\|,
$$

where we use the caracterization of Herz-type Triebel-Lizorkin spaces by local means. Applying Lemma A.4 of [3], see also Lemma 8.3 of [1, we obtain

$$
\inf _{\gamma}(f)_{\min \left(q, \frac{n}{\frac{n}{q}+\alpha}, \beta\right), \gamma}^{*} \approx \sup (f)_{\min \left(q, \frac{n}{\frac{n}{q}+\alpha}, \beta\right), \gamma}^{*}
$$

Hence for $\gamma>0$ sufficiently large we obtain by applying Lemma 2,

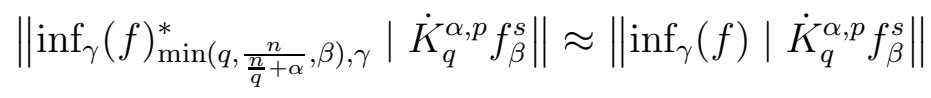


and

$$
\left\|\sup (f)_{\min \left(q, \frac{n}{\frac{n}{q}+\alpha}, \beta\right), \gamma}^{*}\left|\dot{K}_{q}^{\alpha, p} f_{\beta}^{s}\|\approx\| \sup (f)\right| \dot{K}_{q}^{\alpha, p} f_{\beta}^{s}\right\|
$$

for any $s \in \mathbb{R}, 0<p, q<\infty, 0<\beta \leq \infty$ and $\alpha>-n / q$. Therefore,

$$
\left\|\inf _{\gamma}(f)\left|\dot{K}_{q}^{\alpha, p} f_{\beta}^{s}\|\approx\| f\right| \dot{K}_{q}^{\alpha, p} F_{\beta}^{s}\right\| \approx\left\|\sup (f) \mid \dot{K}_{q}^{\alpha, p} f_{\beta}^{s}\right\|
$$

Use these estimates and repeating the proof of Theorem 2.2 in [3] or Theorem 2.1 in 25] then complete the proof of Theorem 2 .

Remark 2 From these to prove the embeddings

$$
\dot{K}_{q}^{\alpha_{2}, r} F_{\theta}^{s_{2}} \hookrightarrow \dot{K}_{s}^{\alpha_{1}, p} F_{\beta}^{s_{1}}
$$

we need only to prove

$$
\dot{K}_{q}^{\alpha_{2}, r} f_{\theta}^{s_{2}} \hookrightarrow \dot{K}_{s}^{\alpha_{1}, p} f_{\beta}^{s_{1}},
$$

under the same restrictions on parameters $s_{1}, s_{2}, \alpha_{1}, \alpha_{2}, s, p, q, \beta, r, \theta$.

We end this section with one more lemma, which is basically a consequence of Hardy's inequality in the sequence Lebesgue space $\ell_{q}$.

Lemma 3 Let $0<a<1$ and $0<q \leq \infty$. Let $\left\{\varepsilon_{k}\right\}$ be a sequences of positive real numbers and denote $\delta_{k}=\sum_{j=0}^{k} a^{k-j} \varepsilon_{j}$ and $\eta_{k}=\sum_{j=k}^{\infty} a^{j-k} \varepsilon_{j}, k \in \mathbb{N}_{0}$. Then there exists constant $c>0$ depending only on a and $q$ such that

$$
\left(\sum_{k=0}^{\infty} \delta_{k}^{q}\right)^{1 / q}+\left(\sum_{k=0}^{\infty} \eta_{k}^{q}\right)^{1 / q} \leq c\left(\sum_{k=0}^{\infty} \varepsilon_{k}^{q}\right)^{1 / q} .
$$

\section{Sobolev embeddings for $\dot{K}_{q}^{\alpha, p} F_{\beta}^{s}$ spaces}

It is well-known that

$$
F_{q, \infty}^{s_{2}} \hookrightarrow F_{s, \beta}^{s_{1}}
$$

if $s_{1}-n / s=s_{2}-n / q$, where $0<q<s<\infty$ and $0<\beta \leq \infty$ (see e.g. [14, Theorem 2.7.1]). In this section we generalize these embeddings to Herz-type Triebel-Lizorkin spaces. We need the Sobolev embeddings properties of the above sequence spaces.

Theorem 3 Let $\alpha_{1}, \alpha_{2}, s_{1}, s_{2} \in \mathbb{R}, 0<s, q<\infty, 0<r \leq p<\infty, 0<\beta \leq \infty, \alpha_{1}>$ $-n / s$ and $\alpha_{2}>-n / q$. We suppose that

$$
s_{1}-n / s-\alpha_{1}=s_{2}-n / q-\alpha_{2} .
$$

Let $0<q<s<\infty$ and $\alpha_{2} \geq \alpha_{1}$ or $0<s \leq q<\infty$ and

$$
\alpha_{2}+n / q \geq \alpha_{1}+n / s \text {. }
$$

Then

$$
\dot{K}_{q}^{\alpha_{2}, r} f_{\theta}^{s_{2}} \hookrightarrow \dot{K}_{s}^{\alpha_{1}, p} f_{\beta}^{s_{1}}
$$

where

$$
\theta=\left\{\begin{array}{ccc}
\beta & \text { if } & 0<s \leq q<\infty \\
\infty & \text { and } \alpha_{2}+n / q=\alpha_{1}+n / s \\
\text { otherwise }
\end{array}\right.
$$


Proof. We would like to mention that this embedding was proved in 2, Theorem 5.9] under the restriction $\max \left(0, \frac{\alpha_{1} s}{q}\right) \leq \alpha_{2} \leq\left(\alpha_{1}+\frac{n}{s}\right) \frac{r}{p}-\frac{n}{q}$. Here we use a different method to omit this condition.

Step 1. Let us prove that $0<r \leq p<\infty$ is necessary. In the calculations below we consider the 1-dimensional case for simplicity. For any $v \in \mathbb{N}_{0}$ and $N \geq 2$, we put

$$
\lambda_{v, m}^{N}=\left\{\begin{array}{ccc}
2^{-\left(s_{1}-\frac{1}{s}-\alpha_{1}\right) v} \sum_{i=2}^{N-2} \chi_{i}\left(2^{v-1}\right) & \text { if } & \begin{array}{c}
m=1 \\
0
\end{array} \\
& \text { otherwise }
\end{array}\right.
$$

$\lambda^{N}=\left\{\lambda_{v, m}^{N}: v \in \mathbb{N}_{0}, m \in \mathbb{Z}\right\}$. We have

$$
\left\|\lambda^{N} \mid \dot{K}_{s}^{\alpha_{1}, p} f_{\beta}^{s_{1}}\right\|^{p}=\sum_{k=-\infty}^{\infty} 2^{\alpha_{1} k p}\left\|\left(\sum_{v=0}^{\infty} \sum_{m \in \mathbb{Z}} 2^{v s_{1} \beta}\left|\lambda_{v, m}^{N}\right|^{\beta} \chi_{v, m}\right)^{1 / \beta} \chi_{k}\right\|_{s}^{p} .
$$

We can rewrite the last statement as follows:

$$
\begin{aligned}
& \sum_{k=1-N}^{0} 2^{\alpha_{1} k p}\left\|\left(\sum_{v=2}^{N-2} 2^{\left(\frac{1}{s}+\alpha_{1}\right) v \beta} \chi_{v, 1}\right)^{1 / \beta} \chi_{k}\right\|_{s}^{p} \\
= & \sum_{k=1-N}^{0} 2^{\alpha_{1} k p}\left\|2^{\left(\frac{1}{s}+\alpha_{1}\right)(1-k)} \chi_{1-k, 1}\right\|_{s}^{p}=c N,
\end{aligned}
$$

where the constant $c>0$ does not depend on $N$. Now

$$
\left\|\lambda^{N} \mid \dot{K}_{q}^{\alpha_{2}, r} f_{\theta}^{s_{2}}\right\|^{r}=\sum_{k=-\infty}^{\infty} 2^{\alpha_{2} k r}\left\|\left(\sum_{v=0}^{\infty} 2^{v s_{2} \theta}\left|\lambda_{v, 1}^{N}\right|^{\theta} \chi_{v, 1}\right)^{1 / \theta} \chi_{k}\right\|_{q}^{r} .
$$

Again we can rewrite the last statement as follows:

$$
\begin{aligned}
& \sum_{k=1-N}^{0} 2^{\alpha_{2} k r}\left\|\left(\sum_{v=2}^{N-2} 2^{\left(s_{2}-s_{1}+\frac{1}{s}+\alpha_{1}\right) v \theta} \chi_{v, 1}\right)^{1 / \theta} \chi_{k}\right\|_{q}^{r} \\
= & \sum_{k=1-N}^{0} 2^{\alpha_{2} k r}\left\|2^{\left(s_{2}-s_{1}+\frac{1}{s}+\alpha_{1}\right)(1-k)} \chi_{1-k, 1}\right\|_{q}^{r}=c N,
\end{aligned}
$$

where the constant $c>0$ does not depend on $N$. If the embeddings (14) holds then for any $N \in \mathbb{N}, N^{\frac{1}{p}-\frac{1}{r}} \leq C$. Thus, we conclude that $0<r \leq p<\infty$ must necessarily hold by letting $N \rightarrow+\infty$.

Step 2. We consider the sufficiency of the conditions. First we consider $0<q<s<\infty$ and $\alpha_{2} \geq \alpha_{1}$. In view of the embedding $\ell_{r} \hookrightarrow \ell_{p}$, it is sufficient to prove that

$$
\dot{K}_{q}^{\alpha_{2}, r} f_{\infty}^{s_{2}} \hookrightarrow \dot{K}_{s}^{\alpha_{1}, r} f_{\beta}^{s_{1}} .
$$

By similarity, we only consider the case $\beta=1$. Let $\lambda \in \dot{K}_{q}^{\alpha_{2}, r} f_{\infty}^{s_{2}}$. We have

$$
\begin{aligned}
\left\|\lambda \mid \dot{K}_{s}^{\alpha_{1}, r} f_{1}^{s_{1}}\right\| \leq & \left(\sum_{k=-\infty}^{c_{n}+1} 2^{k \alpha_{1} r}\left\|\sum_{v=0}^{\infty} 2^{v s_{1}} \sum_{m \in \mathbb{Z}^{n}} \lambda_{v, m} \chi_{v, m} \chi_{k}\right\|_{s}^{r}\right)^{1 / r} \\
& +\left(\sum_{k=c_{n}+2}^{\infty} 2^{k \alpha_{1} r}\left\|\sum_{v=0}^{\infty} 2^{v s_{1}} \sum_{m \in \mathbb{Z}^{n}} \lambda_{v, m} \chi_{v, m} \chi_{k}\right\|_{s}^{r}\right)^{1 / r} .
\end{aligned}
$$


Here $c_{n}=1+\left[\log _{2}(2 \sqrt{n}+1)\right]$. The first term can be estimated by

$$
\begin{aligned}
& c\left(\sum_{k=-\infty}^{c_{n}+1} 2^{k \alpha_{1} r}\left\|\sum_{v=0}^{c_{n}-k+1} 2^{v s_{1}} \sum_{m \in \mathbb{Z}^{n}} \lambda_{v, m} \chi_{v, m} \chi_{k}\right\|_{s}^{r}\right)^{1 / r} \\
& +c\left(\sum_{k=-\infty}^{c_{n}+1} 2^{k \alpha_{1} r}\left\|\sum_{v=c_{n}-k+2}^{\infty} 2^{v s_{1}} \sum_{m \in \mathbb{Z}^{n}} \lambda_{v, m} \chi_{v, m} \chi_{k}\right\|_{s}^{r}\right)^{1 / r} \\
= & I+I I .
\end{aligned}
$$

Estimation of $I$. Let $x \in C_{k} \cap Q_{v, m}$ and $y \in Q_{v, m}$. We have $|x-y| \leq 2 \sqrt{n} 2^{-v}<2^{c_{n}-v}$ and from this it follows that $|y|<2^{c_{n}-v}+2^{k} \leq 2^{c_{n}-v+2}$, which implies that $y$ is located in some ball $B\left(0,2^{c_{n}-v+2}\right)$. Then

$$
\left|\lambda_{v, m}\right|^{t}=2^{n v} \int_{\mathbb{R}^{n}}\left|\lambda_{v, m}\right|^{t} \chi_{v, m}(y) d y \leq 2^{n v} \int_{B\left(0,2^{c_{n}-v+2}\right)}\left|\lambda_{v, m}\right|^{t} \chi_{v, m}(y) d y,
$$

if $x \in C_{k} \cap Q_{v, m}$ and $t>0$. Therefore for any $x \in C_{k}$

$$
\begin{aligned}
\sum_{m \in \mathbb{Z}^{n}}\left|\lambda_{v, m}\right|^{t} \chi_{v, m}(x) & \leq 2^{n v} \int_{B\left(0,2^{c_{n}-v+2}\right)} \sum_{m \in \mathbb{Z}^{n}}\left|\lambda_{v, m}\right|^{t} \chi_{v, m}(y) d y \\
& =2^{n v}\left\|\sum_{m \in \mathbb{Z}^{n}}\left|\lambda_{v, m}\right| \chi_{v, m} \chi_{B\left(0,2^{c_{n}-v+2}\right)}\right\|_{t}^{t}
\end{aligned}
$$

Hence

$$
\begin{aligned}
& 2^{\alpha_{1} k}\left\|\sum_{v=0}^{c_{n}-k+1} 2^{v s_{1}} \sum_{m \in \mathbb{Z}^{n}} \lambda_{v, m} \chi_{v, m} \chi_{k}\right\|_{s} \\
\leq & c 2^{\left(\alpha_{1}+\frac{n}{s}\right) k} \sum_{v=0}^{c_{n}-k+1} 2^{v\left(s_{1}+\frac{n}{t}\right)}\left\|\sum_{m \in \mathbb{Z}^{n}}\left|\lambda_{v, m}\right| \chi_{v, m} \chi_{B\left(0,2^{c_{n}-v+2}\right)}\right\|_{t}
\end{aligned}
$$

We may choose $t>0$ such that $\frac{1}{t}>\max \left(\frac{1}{q}, \frac{1}{q}+\frac{\alpha_{2}}{n}\right)$. Using (12) and Lemma 3 to estimate $I^{r}$ by

$$
\begin{aligned}
& c \sum_{v=0}^{\infty} 2^{v\left(s_{2}-\frac{n}{q}-\alpha_{2}+\frac{n}{t}\right) r}\left\|\sum_{m \in \mathbb{Z}^{n}}\left|\lambda_{v, m}\right| \chi_{v, m} \chi_{B\left(0,2^{c} n-v+2\right.}\right\|_{t}^{r} \\
\leq & c \sum_{v=0}^{\infty} 2^{v\left(s_{2}-\frac{n}{q}-\alpha_{2}+\frac{n}{t}\right) r}\left(\sum_{i \leq-v}\left\|\sum_{m \in \mathbb{Z}^{n}}\left|\lambda_{v, m}\right| \chi_{v, m} \chi_{i+c_{n}+2}\right\|_{t}^{\sigma}\right)^{r / \sigma} \\
\leq & c \sum_{v=0}^{\infty} 2^{v \frac{n r}{d}}\left(\sum_{i \leq-v} 2^{i \frac{n \sigma}{d}+\alpha_{2} \sigma i}\left\|\sup _{j} \sum_{m \in \mathbb{Z}^{n}} 2^{s_{2} j}\left|\lambda_{j, m}\right| \chi_{j, m} \chi_{i+c_{n}+2}\right\|_{q}^{\sigma}\right)^{r / \sigma},
\end{aligned}
$$

by Hölder's inequality, with $\sigma=\min (1, t)$ and $\frac{n}{d}=\frac{n}{t}-\frac{n}{q}-\alpha_{2}$. Again, we apply Lemma 3) to obtain

$$
I^{r} \leq c \sum_{i=0}^{\infty} 2^{-\alpha_{2} i r}\left\|\sup _{j} \sum_{m \in \mathbb{Z}^{n}} 2^{s_{2} j}\left|\lambda_{j, m}\right| \chi_{j, m} \chi_{-i+c_{n}+2}\right\|_{q}^{r} \leq c\left\|\lambda \mid \dot{K}_{q}^{\alpha_{2}, r} f_{\infty}^{s_{2}}\right\|^{r} .
$$


Estimation of $I I$. We see that it suffices to show that for any $k \leq c_{n}+1$

$$
\begin{aligned}
2^{k \alpha_{1}}\left\|\sum_{v=c_{n}-k+2}^{\infty} 2^{v s_{1}} \sum_{m \in \mathbb{Z}^{n}} \lambda_{v, m} \chi_{v, m} \chi_{k}\right\|_{s} & \leq C^{s / q} 2^{k \alpha_{2}}\left\|_{v \geq c_{n}-k+2} \sum_{m \in \mathbb{Z}^{n}} 2^{v s_{2}} \lambda_{v, m} \chi_{v, m} \chi_{\widetilde{C}_{k}}\right\|_{q} \\
& =\delta,
\end{aligned}
$$

where $\widetilde{C}_{k}=\left\{x \in \mathbb{R}^{n}: 2^{k-2}<|x|<2^{k+2}\right\}$ and $C=2 \max \left(\frac{2^{\frac{1}{q}}}{1-2^{\frac{n}{s}-\frac{n}{q}}}, \frac{2^{\frac{n}{s}}}{2^{\frac{n}{s}}-1}\right)$. This claim can be reformulated as showing that

$$
\int_{C_{k}} 2^{k \alpha_{1} s} \delta^{-s}\left(\sum_{v=c_{n}-k+2}^{\infty} 2^{v s_{1}} \sum_{m \in \mathbb{Z}^{n}}\left|\lambda_{v, m}\right| \chi_{v, m}(x)\right)^{s} d x \leq 1 .
$$

The left-hand side can be rewritten us

$$
\begin{aligned}
& \int_{C_{k}} \delta^{-s}\left(\sum_{v=c_{n}-k+2}^{\infty} 2^{v\left(\frac{n}{s}-\frac{n}{q}\right)+\left(\alpha_{1}-\alpha_{2}\right)(v+k)+v s_{2}+k \alpha_{2}} \sum_{m \in \mathbb{Z}^{n}}\left|\lambda_{v, m}\right| \chi_{v, m}(x)\right)^{s} d x \\
\leq & \int_{C_{k}} \delta^{-s}\left(\sum_{v=c_{n}-k+2}^{\infty} 2^{v\left(\frac{n}{s}-\frac{n}{q}\right)+v s_{2}+k \alpha_{2}} \sum_{m \in \mathbb{Z}^{n}}\left|\lambda_{v, m}\right| \chi_{v, m}(x)\right)^{s} d x=T_{k},
\end{aligned}
$$

since $\alpha_{2} \geq \alpha_{1}$. Let us prove that $T_{k} \leq 1$ for any $k \leq c_{n}+1$. Our estimate use partially some decomposition techniques already used in [18].

Case 1. $\sup _{v \geq c_{n}-k+2, m} 2^{v s_{2}+k \alpha_{2}}\left|\lambda_{v, m}\right| \chi_{v, m}(x) \leq \delta$. In this case we obtain

$$
T_{k} \leq C^{s} \int_{C_{k}}\left(\delta^{-1} \sup _{v \geq c_{n}-k+2} \sum_{m \in \mathbb{Z}^{n}} 2^{v s_{2}+k \alpha_{2}}\left|\lambda_{v, m}\right| \chi_{v, m}(x)\right)^{q} d x \leq 1 .
$$

Case 2. $\sup _{v \geq c_{n}-k+2, m} 2^{v s_{2}+k \alpha_{2}}\left|\lambda_{v, m}\right| \chi_{v, m}(x)>\delta$. We can distinguish two cases as follows:

- $\delta^{-1} \sup _{v \geq c_{n}-k+2, m} 2^{v s_{2}+k \alpha_{2}}\left|\lambda_{v, m}\right| \chi_{v, m}(x)=\infty$, then there is nothing to prove.

- $\delta<\sup _{v \geq c_{n}-k+2, m} 2^{v s_{2}+k \alpha_{2}}\left|\lambda_{v, m}\right| \chi_{v, m}(x)<\infty$. Let $N \in \mathbb{N}$ be such that

$$
2^{\frac{n N}{q}}<\delta^{-1} \sup _{v \geq c_{n}-k+2} \sum_{m \in \mathbb{Z}^{n}} 2^{v s_{2}+k \alpha_{2}}\left|\lambda_{v, m}\right| \chi_{v, m}(x)<2^{\frac{(N+1) n}{q}} .
$$

Subcase 2.1. $k \geq c_{n}-N+2$. We split the sum over $v \geq c_{n}-k+2$ into two parts,

$$
\sum_{v=c_{n}-k+2}^{\infty} \cdots=\sum_{v=c_{n}-k+2}^{N} \cdots+\sum_{v=N+1}^{\infty} \cdots
$$

Let $x \in C_{k} \cap Q_{v, m}$ and $y \in Q_{v, m}$. We have $|x-y| \leq 2 \sqrt{n} 2^{-v}<2^{c_{n}-v}$ and from this it follows that $2^{k-2}<|y|<2^{c_{n}-v}+2^{k}<2^{k+2}$, which implies that $y$ is located in $\widetilde{C}_{k}$. Then

$$
\left|\lambda_{v, m}\right|^{q}=2^{n v} \int_{\mathbb{R}^{n}}\left|\lambda_{v, m}\right|^{q} \chi_{v, m}(y) d y \leq 2^{n v} \int_{\widetilde{C}_{k}}\left|\lambda_{v, m}\right|^{q} \chi_{v, m}(y) d y,
$$

if $x \in C_{k} \cap Q_{v, m}$. But this immediately implies that

$$
\begin{aligned}
\sum_{m \in \mathbb{Z}^{n}}\left|\lambda_{v, m}\right|{ }^{q} \chi_{v, m}(x) & \leq 2^{n v} \int_{\widetilde{C}_{k}} \sum_{m \in \mathbb{Z}^{n}}\left|\lambda_{v, m}\right|^{q} \chi_{v, m}(y) d y \\
& =2^{n v}\left\|\sum_{m \in \mathbb{Z}^{n}}\left|\lambda_{v, m}\right| \chi_{v, m} \chi_{\widetilde{C}_{k}}\right\|_{q}^{q} \leq 2^{\left(\frac{n}{q}-s_{2}\right) q v-\alpha_{2} q k} \delta^{q} .
\end{aligned}
$$


Therefore for any $x \in C_{k}$

$$
\delta^{-1} \sum_{v=c_{n}-k+2}^{N} 2^{v\left(\frac{n}{s}-\frac{n}{q}\right)+v s_{2}+k \alpha_{2}} \sum_{m \in \mathbb{Z}^{n}}\left|\lambda_{v, m}\right| \chi_{v, m}(x) \leq \sum_{v=c_{n}-k}^{N} 2^{\frac{n}{s} v} \leq C 2^{\frac{n}{s} N}
$$

and

$$
\begin{aligned}
& \delta^{-1} \sum_{v=N+1}^{\infty} 2^{v\left(\frac{n}{s}-\frac{n}{q}\right)+v s_{2}+k \alpha_{2}} \sum_{m \in \mathbb{Z}^{n}}\left|\lambda_{v, m}\right| \chi_{v, m}(x) \\
= & 2^{\left(\frac{n}{s}-\frac{n}{q}\right) N} \delta^{-1} \sum_{v=N+1}^{\infty} 2^{(v-N)\left(\frac{n}{s}-\frac{n}{q}\right)+v s_{2}+k \alpha_{2}} \sum_{m \in \mathbb{Z}^{n}}\left|\lambda_{v, m}\right| \chi_{v, m}(x) \\
\leq & c 2^{\left(\frac{n}{s}-\frac{n}{q}\right) N} \delta^{-1} \sup _{v \geq c_{n}-k+2} \sum_{m \in \mathbb{Z}^{n}} 2^{v s_{2}+k \alpha_{2}}\left|\lambda_{v, m}\right| \chi_{v, m}(x) \\
\leq & C 2^{\frac{n}{s} N} .
\end{aligned}
$$

Hence

$$
T_{k} \leq C^{s} \int_{C_{k}} 2^{n N} d x \leq C^{s} \int_{C_{k}}\left(\delta^{-1} \sup _{v \geq c_{n}-k+2} \sum_{m \in \mathbb{Z}^{n}} 2^{v s_{2}+k \alpha_{2}}\left|\lambda_{v, m}\right| \chi_{v, m}(x)\right)^{q} d x \leq 1 .
$$

Subcase 2.2. $k<c_{n}-N+2$. We use the same of arguments as in Subcase 2.1, in view of the fact that $\sum_{v=c_{n}-k+2}^{\infty} \cdots \leq \sum_{v=N+1}^{\infty} \cdots$.

Estimate of (15). The arguments here are quite similar to those used in the estimation of $I I$. This complete the proof of the first case.

Now we consider the case $0<s \leq q<\infty$ and $\alpha_{2}+n / q>\alpha_{1}+n / s$. We only need to estimate the part $T_{k}$. Hölder's inequality implies that

$$
\begin{aligned}
T_{k} & \leq\left\|\delta^{-1} \sum_{v=c_{n}-k+2}^{\infty} 2^{\left(\frac{n}{s}-\frac{n}{q}+\alpha_{1}-\alpha_{2}\right)(v+k)} 2^{v s_{2}+k \alpha_{2}} \sum_{m \in \mathbb{Z}^{n}}\left|\lambda_{v, m}\right| \chi_{v, m} \chi_{k}\right\|_{q}^{s} \\
& \leq\left\|\delta^{-1} \sup _{v \geq c_{n}-k+2} \sum_{m \in \mathbb{Z}^{n}} 2^{v s_{2}+k \alpha_{2}}\left|\lambda_{v, m}\right| \chi_{v, m} \chi_{k}\right\|_{q}^{s}=C^{-s / q}
\end{aligned}
$$

where the last inequality follows by the fact that $\alpha_{2}+n / q>\alpha_{1}+n / s$. The remaining case can be easily solved. The proof is complete.

As a corollary of Theorems 2 and 3, we have the following Sobolev embedding for $\dot{K}_{q}^{\alpha, p} F_{\beta}^{s}$ spaces.

Theorem 4 Let $\alpha_{1}, \alpha_{2}, s_{1}, s_{2} \in \mathbb{R}, 0<s, q<\infty, 0<r \leq p<\infty, 0<\beta \leq \infty, \alpha_{1}>$ $-n / s$ and $\alpha_{2}>-n / q$. We suppose that

$$
s_{1}-n / s-\alpha_{1}=s_{2}-n / q-\alpha_{2} .
$$

Let $0<q<s<\infty$ and $\alpha_{2} \geq \alpha_{1}$ or $0<s \leq q<\infty$ and

$$
\alpha_{2}+n / q \geq \alpha_{1}+n / s .
$$

Then

$$
\dot{K}_{q}^{\alpha_{2}, r} F_{\theta}^{s_{2}} \hookrightarrow \dot{K}_{s}^{\alpha_{1}, p} F_{\beta}^{s_{1}},
$$

where

$$
\theta=\left\{\begin{array}{ccc}
\beta & \text { if } & 0<s \leq q<\infty \\
\infty & \text { and } \alpha_{2}+n / q=\alpha_{1}+n / s \\
\text { otherwise }
\end{array}\right.
$$


Remark 3 We would like to mention that (13) and $s_{1}-n / s-\alpha_{1} \leq s_{2}-n / q-\alpha_{2}$ are necessary, see [2].

From Theorem 4 and the fact that $\dot{K}_{s}^{0, s} F_{\beta}^{s_{1}}=F_{s, \beta}^{s_{1}}$ we immediately arrive at the following corollaries.

Corollary 1 Let $s_{1}, s_{2} \in \mathbb{R}, 0<s, q<\infty, s_{1}-n / s=s_{2}-n / q-\alpha_{2}, 0<r \leq s<\infty$ and $0<\beta \leq \infty$. Let $0<q<s<\infty$ and $\alpha_{2} \geq 0$ or $0<s \leq q<\infty$ and $\alpha_{2}+n / q \geq n / s$. Then

$$
\dot{K}_{q}^{\alpha_{2}, r} F_{\theta}^{s_{2}} \hookrightarrow F_{s, \beta}^{s_{1}},
$$

where

$$
\theta=\left\{\begin{array}{ccc}
\beta & \text { if } & 0<s \leq q<\infty \text { and } \alpha_{2}+n / q=n / s \\
\infty & \text { otherwise }
\end{array}\right.
$$

Corollary 2 Let $s_{1}, s_{2} \in \mathbb{R}, 0<s, q<\infty, s_{1}-n / s-\alpha_{1}=s_{2}-n / q, 0<q \leq p<\infty$ and $0<\beta \leq \infty$. Let $0<q<s<\infty$ and $\alpha_{1} \leq 0$ or $0<s \leq q<\infty$ and $n / q \geq \alpha_{1}+n / s$. Then

$$
F_{q, \theta}^{s_{2}} \hookrightarrow \dot{K}_{s}^{\alpha_{1}, p} F_{\beta}^{s_{1}}
$$

where

$$
\theta=\left\{\begin{array}{ccc}
\beta & \text { if } & 0<s \leq q<\infty \text { and } n / q=\alpha_{1}+n / s \\
\infty & \text { otherwise. }
\end{array}\right.
$$

From the above corollaries and the fact that $\dot{K}_{q}^{\alpha, r} F_{2}^{0}=\dot{K}_{q}^{\alpha, r}$ for $1<r, q<\infty$ and $-\frac{n}{q}<\alpha<n-\frac{n}{q}$, see [19] we obtain the following embeddings between Herz and Triebel-Lizorkin spaces

$$
\dot{K}_{q}^{\alpha_{2}, r} \hookrightarrow F_{s, \beta}^{s_{1}},
$$

if $n / s-s_{1}=n / q+\alpha_{2}, 1<r \leq s<\infty, 0<\beta \leq \infty$, and

$$
1<q<s<\infty \quad \text { and } \quad 0 \leq \alpha_{2}<n-\frac{n}{q}
$$

or

$$
1<s \leq q<\infty \quad \text { and } \quad \frac{n}{s}-\frac{n}{q}<\alpha_{2}<n-\frac{n}{q}
$$

or

$$
1<s \leq q<\infty, \alpha_{2}=\frac{n}{s}-\frac{n}{q} \quad \text { and } \quad \beta=2 .
$$

Again we obtain

$$
F_{q, \theta}^{s_{2}} \hookrightarrow \dot{K}_{s}^{\alpha_{1}, p}
$$

holds if $n / s+\alpha_{1}=n / q-s_{2}, 0<\max (q, 1)<p<\infty($ or $1<q=p<\infty), 0<\theta \leq \infty$ and

or

$$
0<\max (q, 1)<s<\infty \text { and }-\frac{n}{s}<\alpha_{1} \leq 0
$$

$$
1<s \leq q<\infty \quad \text { and } \quad-\frac{n}{s}<\alpha_{1}<n / q-n / s
$$

or

$$
1<s \leq q<\infty, \alpha_{1}=n / q-n / s \text { and } \theta=2
$$


From the Jawerth-Franke embeddings we have

$$
F_{t, \infty}^{s_{3}} \hookrightarrow B_{q, t}^{s_{2}} \hookrightarrow F_{s, \beta}^{s_{1}},
$$

if $s_{1}, s_{2}, s_{3} \in \mathbb{R}, s_{1}-n / s=s_{2}-n / q=s_{3}-n / t, 0<t<q<s<\infty$ and $0<\beta \leq \infty$, see [16, p. 60]. Using our results, we have the following useful consequences.

Corollary 3 Let $s_{1}, s_{2}, s_{3} \in \mathbb{R}, 0<s, q, t<\infty, s_{1}-n / s=s_{2}-n / q=s_{3}-n / t$ and $0<\beta \leq \infty$. Then

$$
F_{t, \infty}^{s_{3}} \hookrightarrow \dot{K}_{q}^{0, s} F_{\infty}^{s_{2}} \hookrightarrow F_{s, \beta}^{s_{1}}, \quad 0<t \leq q<s<\infty .
$$

To prove this it is sufficient to take in Corollary 1, $r=s$ and $\alpha_{2}=0$. However the desired embeddings are an immediate consequence of the fact that

$$
F_{t, \infty}^{s_{3}} \hookrightarrow F_{q, \infty}^{s_{2}}=\dot{K}_{q}^{0, q} F_{\infty}^{s_{2}} \hookrightarrow \dot{K}_{q}^{0, s} F_{\infty}^{s_{2}}
$$

Corollary 4 Let $s_{1}, s_{2} \in \mathbb{R}, 0<s, q<\infty, s_{1}-n / s=s_{2}-n / q$ and $0<\beta \leq \infty$. Then

$$
F_{q, \infty}^{s_{2}} \hookrightarrow \dot{K}_{s}^{0, q} F_{\beta}^{s_{1}} \hookrightarrow F_{s, \beta}^{s_{1}}, \quad 0<q<s<\infty .
$$

To prove this it is sufficient to take in Corollary 2, $p=q$ and $\alpha_{1}=0$. Then the desired embeddings are an immediate consequence of the fact that

$$
F_{q, \infty}^{s_{2}} \hookrightarrow \dot{K}_{s}^{0, q} F_{\beta}^{s_{1}} \hookrightarrow \dot{K}_{s}^{0, s} F_{\beta}^{s_{1}}=F_{s, \beta}^{s_{1}}
$$

\section{Applications}

In this section, we give a simple application of Theorems 3 and 4 .

Theorem 5 Let $s \in \mathbb{R}, 0<p, q, \beta<\infty$ and $\alpha>-n / q$. Then there exists a linear isomorphism $T$ which maps $\dot{K}_{q}^{\alpha, p} F_{\beta}^{s}$ onto $\dot{K}_{q}^{\alpha, p} f_{\beta}^{s}$. Moreover, there is an unconditional basis in $\dot{K}_{q}^{\alpha, p} F_{\beta}^{s}$.

The mapping $T$ is generated by an appropriate wavelet system. A proof of this theorem can be found in $\mathrm{Xu}[24$ for the non-homogeneous Herz-type Triebel-Lizorkin spaces and $\alpha>0$. This result is also true for the spaces $\dot{K}_{q}^{\alpha, p} F_{\beta}^{s}$, with $\alpha>-n / q$. Indeed, the problem can be reduced to proof the $\dot{K}_{q}^{\alpha, p} f_{\beta}^{s}$-version of Lemma 3.5 in Xu [24]. Therefore we need to recall the definition of molecules.

Definition 4 Let $K, L \in \mathbb{N}_{0}$ and let $M>0$. A K-times continuously differentiable function $a \in C^{K}\left(\mathbb{R}^{n}\right)$ is called $[K, L, M]$-molecule concentrated in $Q_{v, m}$, if for some $v \in \mathbb{N}_{0}$ and $m \in \mathbb{Z}^{n}$

$$
\left|D^{\alpha} a(x)\right| \leq 2^{v|\alpha|}\left(1+2^{v}\left|x-2^{-v} m\right|\right)^{-M}, \quad \text { for } \quad 0 \leq|\alpha| \leq K, x \in \mathbb{R}^{n}
$$

and if

$$
\int_{\mathbb{R}^{n}} x^{\alpha} a(x) d x=0, \quad \text { for } \quad 0 \leq|\alpha|<L \text { and } v \geq 1 .
$$


If the molecule $a$ is concentrated in $Q_{v, m}$, that means if it fulfills (16) and (17), then we will denote it by $a_{v m}$. For $v=0$ or $L=0$ there are no moment conditions (17) required.

Now, we prove the $\dot{K}_{q}^{\alpha, p} f_{\beta}^{s}$-version of Lemma 3.5 in Xu [24].

Lemma 4 Let $s \in \mathbb{R}, 0<p, q<\infty, 0<\beta \leq \infty$ and $\alpha>-n / q$. Furthermore, let $K, L \in \mathbb{N}_{0}$ and let $M>0$ with

$$
L>n\left(\frac{1}{\min (1, q, \beta)}-1\right)-1-s, \quad K \text { arbitrary and } M \text { large enough. }
$$

If $a_{v m}$ are $[K, L, M]$-molecules concentrated in $Q_{v, m}$ and $\lambda=\left\{\lambda_{v, m} \in \mathbb{C}: v \in \mathbb{N}_{0}, m \in\right.$ $\left.\mathbb{Z}^{n}\right\} \in \dot{K}_{q}^{\alpha, p} f_{\beta}^{s}$, then the sum

$$
\sum_{v=0}^{\infty} \sum_{m \in \mathbb{Z}^{n}} \lambda_{v, m} a_{v m}
$$

converges in $\mathcal{S}^{\prime}\left(\mathbb{R}^{n}\right)$.

Proof. We use the arguments of [16], see also [5]. Let $\varphi \in \mathcal{S}\left(\mathbb{R}^{n}\right)$. We get from the moment conditions (17) for fixed $v \in \mathbb{N}_{0}$

$$
\begin{aligned}
& \int_{\mathbb{R}^{n}} \sum_{m \in \mathbb{Z}^{n}} \lambda_{v, m} a_{v m}(y) \varphi(y) d y \\
= & \int_{\mathbb{R}^{n}} \sum_{m \in \mathbb{Z}^{n}} \lambda_{v, m} 2^{-v(L+1)} a_{v m}(y)\left(\varphi(y)-\sum_{|\beta|<L}\left(y-2^{-v} m\right)^{\beta} \frac{D^{\beta} \varphi\left(2^{-v} m\right)}{\beta !}\right) 2^{v(L+1)} d y \\
= & \sum_{i=-\infty}^{\infty} \int_{C_{i}} \cdots d y,
\end{aligned}
$$

where $C_{i}=\left\{y \in \mathbb{R}^{n}: 2^{i-1} \leq|y|<2^{i}\right\}$ for any $i \in \mathbb{Z}$. Let us estimate the sum $\sum_{i=-\infty}^{0} \cdots$. We use the Taylor expansion of $\varphi$ up to order $L-1$ with respect to the off-points $2^{-v} m$, we obtain

$$
\varphi(y)-\sum_{|\beta|<L}\left(y-2^{-v} m\right)^{\beta} \frac{D^{\beta} \varphi\left(2^{-v} m\right)}{\beta !}=\sum_{|\beta|=L}\left(y-2^{-v} m\right)^{\beta} \frac{D^{\beta} \varphi(\xi)}{\beta !},
$$

with $\xi$ on the line segment joining $y$ and $2^{-v} m$. Since $1+|y| \leq(1+|\xi|)\left(1+\left|y-2^{-v} m\right|\right)$, we estimate

$$
\begin{aligned}
\left|\sum_{|\beta|=L}\left(y-2^{-v} m\right)^{\beta} \frac{D^{\beta} \varphi(\xi)}{\beta !}\right| & \leq\left(1+\left|y-2^{-v} m\right|\right)^{L} \sum_{|\beta|=L} \frac{\left|D^{\beta} \varphi(\xi)\right|}{\beta !} \\
& \leq\left(1+\left|y-2^{-v} m\right|\right)^{L}(1+|\xi|)^{-S}\|\varphi\|_{S, L} \\
& \leq c(1+|y|)^{-S}\left(1+\left|y-2^{-v} m\right|\right)^{L+S}
\end{aligned}
$$


where $S>0$ is at our disposal. Let $0<t<\min (1, q)=1+q-\frac{q}{\min (1, q)}$ and $h=$ $s+\frac{n}{q}(t-1)$ be such that $n\left(1-\frac{1}{\min (1, q)}\right)+s>h>-1-L$. Since $a_{v m}$ are $[K, L, M]$ molecules, then $2^{-v(L+1)}\left|a_{v m}(y)\right| \leq 2^{h v} 2^{-v(L+1+h)}\left(1+2^{v}\left|y-2^{-v} m\right|\right)^{-M}$. Therefore, The sum $\sum_{i=-\infty}^{0} \cdots$ can be estimated by

$$
c 2^{-v(L+1+h)} \sum_{i=-\infty}^{0} \int_{C_{i}} \sum_{m \in \mathbb{Z}^{n}} 2^{h v}\left|\lambda_{v, m}\right|\left(1+2^{v}\left|y-2^{-v} m\right|\right)^{L+S-M}(1+|y|)^{-S} d y .
$$

Since $M$ can be taken large enough, by Lemma 4 in [5] we obtain

$$
\sum_{m \in \mathbb{Z}^{n}}\left|\lambda_{v, m}\right|\left(1+2^{v}\left|y-2^{-v} m\right|\right)^{L+S-M} \leq c \mathcal{M}\left(\sum_{m \in \mathbb{Z}^{n}}\left|\lambda_{v, m}\right| \chi_{v, m}\right)(y)
$$

for any $y \in C_{i} \cap Q_{v, l}$ with $l \in \mathbb{Z}^{n}$. We split $S$ into $R+T$ with $R+\alpha<0$ and $T$ large enough such that $T>\max \left(-R, \frac{n(q-t)}{q}\right)$. Then (19) is bounded by

$$
c 2^{-v(L+1+h)} \sum_{i=-\infty}^{0} 2^{-i R} \int_{C_{i}} \mathcal{M}\left(\sum_{m \in \mathbb{Z}^{n}} 2^{v h}\left|\lambda_{v, m}\right| \chi_{v, m}\right)(y)(1+|y|)^{-T} d y .
$$

Since we have in addition the factor $(1+|y|)^{-T}$, it follows by Hölder's inequality that this expression is bounded by

$$
\begin{aligned}
& c 2^{-v(L+1+h)} \sum_{i=-\infty}^{0} 2^{-i R}\left\|\mathcal{M}\left(\sum_{m \in \mathbb{Z}^{n}} 2^{h v}\left|\lambda_{v, m}\right| \chi_{v, m}\right) \chi_{i}\right\|_{q / t} \\
\leq & c 2^{-v(L+1+h)} \sum_{i=-\infty}^{0} 2^{-i(\alpha+R)}\left\|\sum_{m \in \mathbb{Z}^{n}} 2^{h v}\left|\lambda_{v, m}\right| \chi_{v, m} \mid \dot{K}_{q / t}^{\alpha, \infty}\right\| \\
\leq & c 2^{-v(L+1+h)}\left\|\lambda \mid \dot{K}_{q / t}^{\alpha, p} f_{\infty}^{h}\right\|,
\end{aligned}
$$

where the first inequality follows by the boundedness of the Hardy-Littlewood maximal operator $\mathcal{M}$ on $\dot{K}_{q / t}^{\alpha, \infty}$. Using a combination of the arguments used above, the sum $\sum_{i=1}^{\infty} \ldots$ can be estimated by $c 2^{-v(L+1+h)}\left\|\lambda \mid \dot{K}_{q / t}^{\alpha, p} f_{\infty}^{h}\right\|$. Since $L+1+h>0$, the convergence of (18) is now clear by the embeddings

$$
\dot{K}_{q}^{\alpha, p} f_{\infty}^{s} \hookrightarrow \dot{K}_{q / t}^{\alpha, p} f_{\infty}^{h}
$$

see Theorem 3. The proof is completed.

Let $w$ denote a positive, locally integrable function and $0<p<\infty$. Then the weighted Lebesgue space $L^{p}\left(\mathbb{R}^{n}, w\right)$ contains all measurable functions such that

$$
\left\|f \mid L^{p}\left(\mathbb{R}^{n}, w\right)\right\|=\left(\int_{\mathbb{R}^{n}}|f(x)|^{p} w(x) d x\right)^{1 / p}<\infty .
$$

For $\varrho \in[1, \infty)$ we denote by $\mathcal{A}_{\varrho}$ the Muckenhoupt class of weights, and $\mathcal{A}_{\infty}=\cup_{\varrho \geq 1} \mathcal{A}_{\varrho}$. We refer to [4] for the general properties of these classes. Let $w \in \mathcal{A}_{\infty}, s \in \mathbb{R}$, 
$0<\beta \leq \infty$ and $0<p<\infty$. We define weighted Triebel-Lizorkin spaces $F_{p, q}^{s}\left(\mathbb{R}^{n}, w\right)$ to be the set of all distributions $f \in \mathcal{S}^{\prime}\left(\mathbb{R}^{n}\right)$ such that

$$
\left\|f\left|F_{p, \beta}^{s}\left(\mathbb{R}^{n}, w\right)\|=\|\left(\sum_{j=0}^{\infty} 2^{j s \beta}\left|\mathcal{F}^{-1} \varphi_{j} * f\right|^{\beta}\right)^{1 / \beta}\right| L^{p}\left(\mathbb{R}^{n}, w\right)\right\|
$$

is finite. In the limiting case $q=\infty$ the usual modification is required. The spaces $F_{p, \beta}^{s}\left(\mathbb{R}^{n}, w\right)=F_{p, \beta}^{s}(w)$ are independent of the particular choice of the smooth dyadic resolution of unity $\left\{\varphi_{j}\right\}_{j \in \mathbb{N}_{0}}$ appearing in their definitions. They are quasi-Banach spaces (Banach spaces for $p, q \geq 1$ ), and

$$
\mathcal{S}\left(\mathbb{R}^{n}\right) \hookrightarrow F_{p, \beta}^{s}(w) \hookrightarrow \mathcal{S}^{\prime}\left(\mathbb{R}^{n}\right) .
$$

Moreover, for $w \equiv 1 \in \mathcal{A}_{\infty}$ we obtain the usual (unweighted) Triebel-Lizorkin spaces. Let $w_{\gamma}$ be a power weight, i.e., $w_{\gamma}(x)=|x|^{\gamma}$ with $\gamma>-n$. Then in view of the fact that $L^{p}=\dot{K}_{p}^{0, p}$, we have

$$
\left\|f\left|F_{p, \beta}^{s}\left(w_{\gamma}\right)\|\approx\| f\right| \dot{K}_{p}^{\frac{\gamma}{p}, p} F_{\beta}^{s}\right\| .
$$

Applying Corollary 4 in some particular cases yields the following embeddings, see for the case of .

Corollary 5 Let $s_{1}, s_{2} \in \mathbb{R}, 0<q<s<\infty, 0<\beta \leq \infty$ and $w_{\gamma_{1}}(x)=|x|^{\gamma_{1}}$, $w_{\gamma_{2}}(x)=|x|^{\gamma_{2}}$, with $\gamma_{1}>-n$ and $\gamma_{2}>-n$. We suppose that

$$
s_{1}-\frac{n+\gamma_{1}}{s}=s_{2}-\frac{n+\gamma_{2}}{q}
$$

and

$$
\gamma_{2} / q \geq \gamma_{1} / s
$$

Then

$$
F_{q, \infty}^{s_{2}}\left(w_{\gamma_{2}}\right) \hookrightarrow F_{s, \beta}^{s_{1}}\left(w_{\gamma_{1}}\right)
$$

Remark 4 We refer the reader to the recent paper [6] for further results about Sobolev embeddings for weighted spaces of Besov type where the weight belongs to some Muckenhoupt $\mathcal{A}_{\varrho}$ class. Notice that this results are given in [12, Theorem 1.2] but under the restrictions $1<q<s<\infty, 1 \leq \beta \leq \infty$.

\section{References}

[1] M. Bownik, Anisotropic Triebel-Lizorkin Spaces with Doubling Measures. The Journal of Geometric Analysis. 17(3) (2007), 337-424.

[2] D. Drihem, Embeddings properties on Herz-type Besov and Triebel-Lizorkin spaces. Math. Ineq and Appl. 16(2) (2013), 439-460.

[3] M. Frazier, B. Jawerth, A discrete transform and decomposition of distribution spaces. J. Funct. Anal. 93(1) (1990), 34-170. 
[4] J. Garcia-Cuerva,J.L. Rubio de Francia, Weighted norm inequalities and related topics. In: North-Holland Mathematics Studies, Vol. 116, North-Holland, Amsterdam, 1985.

[5] H. Kempka, Atomic, molecular and wavelet decomposition of 2-microlocal Besov and Triebel-Lizorkin spaces with variable integrability, Funct Approx, 43 (1010), 171-208.

[6] D. D. Haroske, L. Skrzypczak, Entropy and approximation numbers of embeddings of function spaces with Muckenhoupt weights. I. Rev. Mat. Complut. 21(1) (2008),135-177.

[7] E. Hernandez, D. Yang, Interpolation of Herz-type Hardy spaces and applications. Math. Nachr. 42 (1998), 564-581.

[8] X. Li, D. Yang, Boundedness of some sublinear operators on Herz spaces. Illinois. J. Math. 40 (1996), 484-501.

[9] S. Lu, D. Yang, The local versions of $H^{p}\left(\mathbb{R}^{n}\right)$ spaces at the origin. Studia. Math. 116 (1995), 103-131.

[10] S. Lu, D. Yang, The decomposition of the weighted Herz spaces on $\mathbb{R}^{n}$ and its applications. Sci. in. China (Ser.A). 38 (1995), 147-158.

[11] S. Lu, D. Yang, Herz-type Sobolev and Bessel potential spaces and their applications. Sci in China (Ser.A). 40 (1997), 113-129.

[12] M. Meyries, M.C. Veraar, Sharp embedding results for spaces of smooth functions with power weights. Studia. Math. 208(3) (2012), 257-293.

[13] L. Tang, D. Yang, Boundedness of vector-valued operators on weighted Herz spaces. Approx. Th. E its Appl. 16 (2000), 58-70.

[14] H. Triebel, Theory of function spaces. Basel: Birkhäuser, 1983.

[15] H. Triebel, Theory of function spaces II. Basel: Birkhäuser, 1992.

[16] H. Triebel, Fractals and spectra. Birkhäuser, Basel 1997.

[17] Y. Tsutsui, The Navier-Stokes equations and weak Herz spaces, Advances in Differential Equations. 16 (2011) 1049-1085.

[18] J. Vybíral, Sobolev and Jawerth embeddings for spaces with variable smoothness and integrability. Ann. Acad. Sci. Fenn. Math. 34(2) (2009), 529-544.

[19] J. Xu, D. Yang, Applications of Herz-type Triebel-Lizorkin spaces. Acta. Math. Sci (Ser. B). 23 (2003), 328-338.

[20] J. Xu, D. Yang, Herz-type Triebel-Lizorkin spaces, I. Acta. Math. Sci (English Ed.). 21(3) (2005), 643-654.

[21] J. Xu, Some properties on Herz-type Besov spaces (in chinese). J. Hunan. Univ (Natural Sci). 30(5) (2003), 75-78. 
[22] J. Xu, Pointwise multipliers of Herz-type Besov spaces and their applications. Appl. Math. 17(1) (2004), 115-121.

[23] J. Xu. Equivalent norms of Herz-type Besov and Triebel-Lizorkin spaces. J. Funct. Spaces. Appl. 3 (2005), 17-31.

[24] J. Xu, Decompositions of non-homogeneous Herz-type Besov and Triebel-Lizorkin spaces. Sci. China. Math. 57(2) (2014), 315-331.

[25] W. Yuan, W. Sickel, D. Yang, Morrey and Campanato Meet Besov, Lizorkin and Triebel. Lecture Notes in Mathematics, vol. 2005, Springer-Verlag, Berlin 2010. 